\title{
A Survey of Adaptive Gateway Discovery Mechanisms in Heterogeneous Networks
}

\author{
Rafi U Zaman \\ Muffakham Jah College of Engineering and Technology, Hyderabad, India \\ rafi.u.zaman@gmail.com \\ Khaleel ur Rahman Khan \\ Department of Computer Science and Engineering, ACE Engineering College, Hyderabad, India \\ khaleelrkhan@aceec.ac.in \\ A.Venugopal Reddy \\ Department of Computer Science and Engineering, UCE (A), Osmania University, Hyderabad, India \\ avgreddy55@gmail.com
}

\begin{abstract}
The communication range of devices within a mobile ad hoc network is inherently restricted. One way of enhancing the communication range of a mobile ad hoc network, is to interconnect it to a wired network like the internet, thus forming a heterogeneous wired cum wireless network. This interconnection also enables mobile nodes to access internet services, and is achieved through gateways. Mobile nodes in the ad hoc network need to discover and register with a gateway in order to obtain Internet connectivity. Gateway discovery mechanisms called proactive, reactive and hybrid exist to enable mobile nodes to register with internet gateways. In the recent times, few optimizations have been proposed to the existing gateway discovery mechanisms in order to make gateway discovery by mobile nodes efficient. In this paper, we present a detailed survey of the state of the art in gateway discovery and selection mechanisms.
\end{abstract}

Index Terms - Mobile Ad Hoc Network, Heterogeneous Networks, Gateway Discovery Mechanisms, Adaptive Gateway Discovery

\section{INTRODUCTION}

Internet has revolutionized the way people communicate. The advent of mobile devices has greatly eased this communication process further. While the Internet facilitates communication without restriction to geographical area, thereby bringing down physical barriers to communication, a mobile ad hoc network (MANET) [1], which is an impromptu network made up of mobile devices, facilitates people to communicate without the need for pre existing infrastructure. Various routing protocols have been proposed to perform routing in MANETs [2]. The performance comparison of some of the major ad hoc network routing protocols can be found in [3]. The Internet and mobile ad hoc network offer a complimentary set of communication features. The Internet is based on a worldwide infrastructure, whereas the infrastructure-less mobile ad hoc network offers the benefit of communication on the move. By itself, a mobile ad hoc network is of limited use, because, communication can take place only among the devices which are part of the network. For this reason, to let mobile devices within an ad hoc network to communicate with any other device anywhere in the world, the mobile ad hoc network is connected to the Internet, resulting in a heterogeneous internetworking architecture. Several strategies exist which define various architectures for interconnecting mobile ad hoc networks to the Internet. These strategies use Mobile IP [4], which provides single hop wireless Internet connectivity, and extend its functionality to multiple hops. A general architecture of an Internet integrated MANET is shown in fig 1.

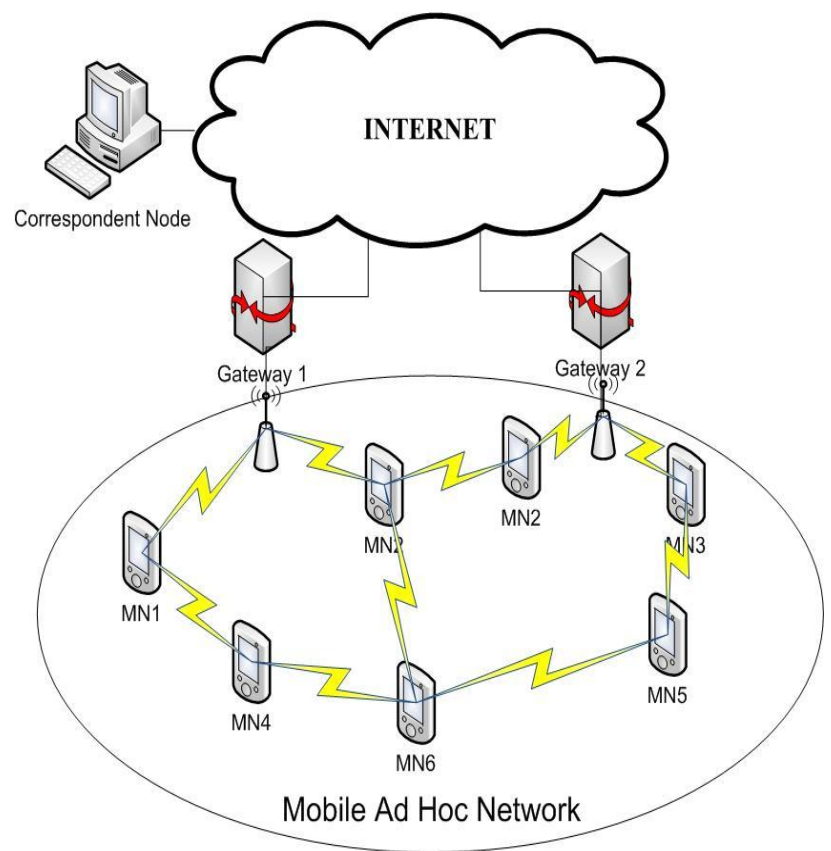

Figure 1. Architecture of an Internet integrated MANET 
For a mobile node to use a gateway, it has to first discover and then register with the gateway. Different gateway discovery and registration mechanisms are described in [5].

One of the important optimizing issues in the interconnection of mobile ad hoc networks with wired Internet is the load balancing of gateways [6]. Other issues are multi homing [7] and hand offs [8]. A frame work for the performance analysis of integration strategies is given in [9].

Gateway registration can be achieved in one of three ways: proactive, reactive or hybrid. An implementation and performance analysis of these three approaches is provided in [10] and [11]. Each of these schemes has its own strengths and drawbacks. The hybrid approach of gateway discovery has attracted the interest of researchers due to its flexibility. Few variations have been proposed to this approach, which are termed adaptive gateway discovery mechanisms.

In this paper, we first discuss the issues in traditional gateway discovery approaches like proactive, reactive and hybrid and then review the adaptive gateway discovery approaches present in the literature. We present the strengths and drawbacks of each of the approaches. Finally, we also discuss the future directions of research in the area of adaptive gateway discovery.

The rest of the paper is structured as follows: section II presents related survey works in the area of heterogeneous networks. Section III contains a brief overview of the three types of traditional gateway discovery mechanisms. Section IV presents the review of adaptive gateway discovery mechanisms. A comparison of the strategies is presented in Section V. Finally, section VI concludes the paper.

\section{RELATED WORK}

A few papers on the survey of strategies for the integration of wired and wireless networks are summarized below.

The work by Abduljalil et al [28] surveys the work integration of mobile IP with mobile ad hoc networks. In [29], [30] and [31], various strategies for integrating mobile ad hoc networks with the internet and the issues to be addressed are discussed. The issue of gateway load balancing in internet integrated MANET has also been addressed extensively in the literature and is surveyed in [6]. An analytical model for the analysis of integration strategies is presented in [32].

\section{GATEWAy Discovery IN HETEROGENEOUS NETWORKS}

As mentioned in section I, mobile nodes need to discover and then register with gateways in order to obtain Internet connectivity. There are three different gateway discovery mechanisms defined in the literature [11]. We present a brief overview of each of these:

\section{A. Proactive Gateway Discovery:}

In the proactive gateway discovery approach, the gateway periodically transmits gateway advertisement messages $\left(\mathrm{GW} \_\mathrm{ADV}\right)$ containing relevant information using which a mobile node can register with the gateway. The GW_ADV message contains a time to live (TTL) field which determines for how many hops this message is forwarded. At each hop, TTL is decreased. Finally, when TTL reaches 0 , the message is not further forwarded. Mobile nodes outside the range of TTL will not be able to use this gateway to obtain internet connectivity.

\section{B. Reactive Gateway Discovery}

In the reactive gateway discovery approach, the gateway does not send GW_ADV messages. Whenever a mobile node desires Internet connectivity, it broadcasts a gateway solicitation message (GW_SOL). When the GW_SOL message reaches a gateway, it sends a GW_ADV message to the mobile node, which then registers with the gateway.

\section{Hybrid Gateway Discovery}

In the hybrid approach, mobile nodes in a part of the mobile ad hoc network, defined by the TTL value use the proactive approach for gateway discovery and the rest of the nodes outside the TTL range use the reactive approach.

For example, in fig 2, TTL is set to 2. When gateway 1 transmits a GW_ADV message, only mobile node 1 ( $M N$ 1) receives this message since it is within the transmission range of the gateway. This is default Mobile IP [4] functionality. On receiving the GW_ADV message, it decrements the TTL value from 2 to 1 and forwards it. Mobile node MN 4 receives the forwarded GW_ADV message. This mobile node again decrements the TTL value, which now becomes 0 . Therefore, the GW_ADV is not further forwarded. Now, mobile node MN 6 which is outside the proactive zone will use the reactive approach of gateway discovery if it desires Internet connectivity. It is said to be in the reactive zone. The same approach is followed by the nodes registered with gateway 2 .

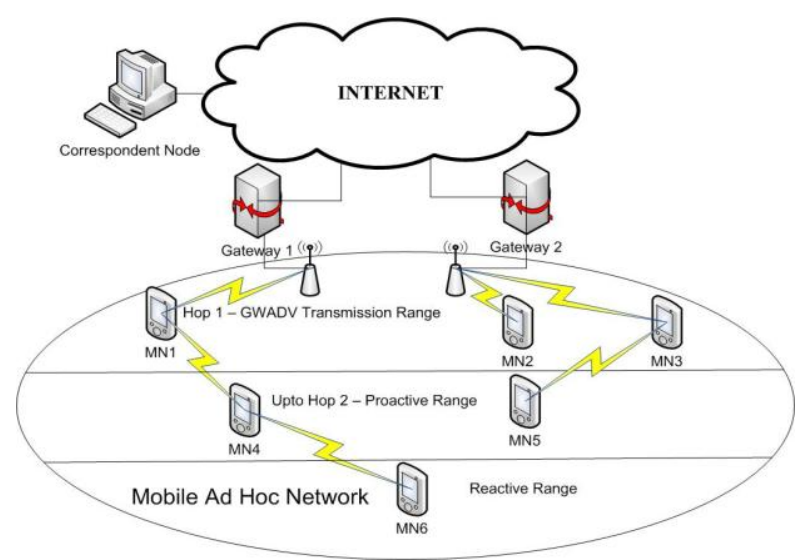

Figure 2. Gateway Discovery Mechanisms in Heterogeneous Networks. 


\section{Issues in Hybrid Gateway Discovery}

The primary challenge in the design of Hybrid Gateway Discovery is how to determine an optimal proactive area. If the proactive area is large (i.e. a large TTL value) more overhead is incurred in maintaining routes in a wider area. On the other hand, if proactive area is small, then less maintenance overhead is incurred but more delay in Gateway Discovery is experienced. In traditional Hybrid Gateway Discovery approach, the TTL is set statically and never changed. This leads to a rigid implementation of the proactive area. Existing integration strategies which use the hybrid approach set the proactive area statically and do not dynamically adjust it, which may not turn out to be a proper range anymore for changing network conditions.

A second issue is determining the periodicity of the GW_ADV messages. The periodicity of the GW_ADV message determines the number of GW_ADV messages that will be flooded into the MANET per second. A high periodicity leads to more control messages in a sparse MANET whereas low periodicity may result in starvation of Internet connectivity to mobile nodes in a dense MANET.

\section{ADAPtive Gateway Discovery Mechanisms IN HETEROGENEOUS NETWORKS}

From the discussion in section III it can be concluded that: To offset changing ad hoc network conditions like node density, transmission quality etc; a static TTL value cannot be used. A modified Hybrid Gateway Discovery mechanism which dynamically adjusts value of TTL and periodicity of GW_ADV messages depending on the MANET characteristics in order to achieve a good trade-off between performance and network overhead is called an Adaptive Gateway Discovery Mechanism. Several novel strategies have been proposed recently which implement adaptive gateway discovery mechanisms in different ways. The purpose of this survey is to review these adaptive gateway discovery mechanisms.

We follow the following approach in reviewing each of the strategies: first we present the salient features of the adaptive gateway discovery mechanism. Then we present the two important features of that adaptive gateway discovery mechanism, viz. TTL value calculation method and GW_ADV periodicity calculation method.

\section{Strategy 1:}

This proposal [12] focuses on dynamic adjustment of the TTL value. An active source is the mobile node which is registered with this gateway and is sending data packets to a correspondent node via this gateway. In the proposed approach, each gateway keeps track of the number of hops at which each of its active sources is located. This is easy for the gateway to do since all data packets of mobile nodes registered with a particular gateway have to pass through that gateway.

To determine the TTL value if the next GW_ADV message, the maximal source coverage algorithm is used. The maximal source coverage algorithm states that the TTL value of the next GW_ADV message of a gateway will be equal to the maximum number of hops among all its active sources.

In fig 3, MN 5 and MN 6 are active nodes. The current value of TTL is 2 . If a static approach of setting the TTL value is used, then TTL will continue to be 2 . On the other hand, in the maximal source coverage approach, the gateway keeps track of the number of hops the active sources are away from it. MN 5 is 2 hops away and MN 6 is 3 hops away. For the next GW_ADV message, the TTL value is set to 3, which is the maximum distance any active source is from the gateway. In this way, the proactive zone is dynamically adjusted and MN 6 also comes within the proactive zone.

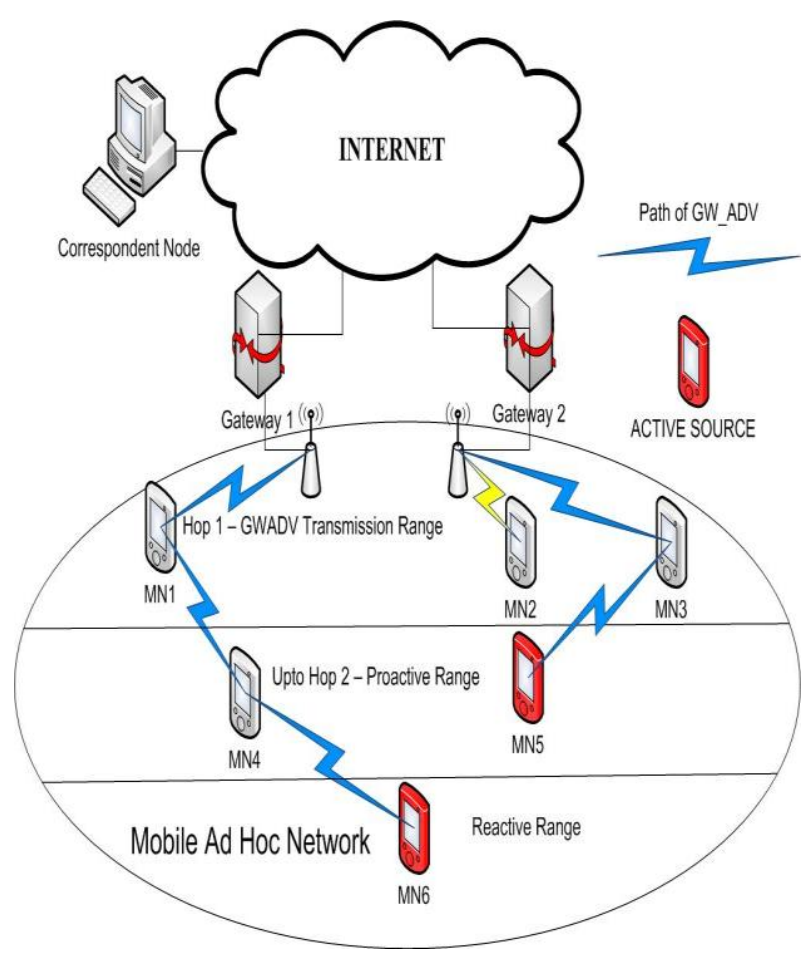

Figure 3. Maximal Source Coverage Adaptive Gateway Discovery

This is one of the first adaptive gateway discovery mechanisms to be proposed. While this mechanism focuses on dynamic adjustment of TTL value, it does not address the issue of periodicity of the GW_ADV messages. The authors have also suggested various other metrics apart from maximal source coverage, like average source coverage and minimal source coverage.

Strategy 2:

This strategy [13] is based on the dynamic adjustment of the TTL value of GW_ADV messages.

An algorithm called maximal benefit coverage is introduced wherein gateways set the TTL value of their 
GW_ADV messages which gives maximal benefit. In order to compute maximal benefit, a ratio is used whose numerator represents the cost of flooding the whole ad hoc network with GW_ADV messages and the denominator denotes the cost of providing internet connectivity without sending a single GW_ADV message. This ratio is used to determine the most appropriate TTL value for the GW_ADV message.

The periodicity of the GW_ADV message is not adjusted dynamically.

\section{Strategy 3:}

A very simple approach is followed in [14]. When an Internet gateway boots up, it starts sending GW_ADV messages in a predefined proactive zone. When the MANET has stabilized, and mobile nodes register with the gateway, it periodically checks the distribution of mobile nodes in the MANET according to simple criteria and adjusts the value of the TTL accordingly. If the average number of hops to all the mobile nodes which desire Internet connectivity is less than 3, the gateway stops sending the periodic GW_ADV message and instead relies on GW_SOL messages from the mobile nodes to provide Internet connectivity. If the average number of hops is greater than 3 but less than 8 , the TTL value of the GW_ADV message is set to the average number of hops. Finally, if the average number of hops is greater than 8 , the TTL value is set to the average number of hops and the regularity of GW_ADV messages is also increased.

\section{Strategy 4:}

This strategy [15] dynamically adjusts the TTL value of the GW_ADV messages as well as the advertisement interval according to the topology change and the movement of nodes.

Consider the case where only one gateway exists and is placed in the center of the MANET and mobile nodes which desire Internet connectivity are uniformly distributed. The ratio of the number of nodes whose distance is less than or equal to $i$ hops from the gateway and the number of nodes whose distance is exactly $\mathrm{i}$ hops from the gateway is estimated and denoted as $\mathrm{P}_{\mathrm{s}}(\mathrm{i})$.

Similarly, when there is more than one gateway with a similar uniform distribution, the ratio of the number of nodes whose distance is less than or equal to i hops from the gateway and the number of nodes whose distance is exactly $i$ hops from the gateway is estimated and denoted as $\mathrm{P}_{\mathrm{m}}(\mathrm{i})$. The average of $\mathrm{P}_{\mathrm{s}}(\mathrm{i})$ and $\mathrm{P}_{\mathrm{m}}(\mathrm{i})$ is used as a threshold value to determine the TTL.

The adjustment of the advertisement periodicity or advertisement interval is based on movement degree, which is defined as the ratio of number of nodes sending GW_SOL messages to the gateway to the number of nodes registered with the gateway during the last GW_ADV cycle. The movement degree is denoted by MD. If the computed MD value is greater than the threshold $\beta$ then the gateway should decrease the advertisement interval, otherwise, the interval should be increased.

\section{Strategy 5:}

Generally, in the MANET routing protocols, bidirectional links are assumed. It is more likely that links will be unidirectional in wireless networks owing to the inherent challenges of the wireless medium. This strategy [16] removes unidirectional links from route computations and redundant broadcast of control packets is inhibited.

For this purpose, a concept of symmetric and asymmetric neighbor sets is used. To dynamically adjust the TTL value of the GW_ADV message, every gateway uses the following statistics: total_nbr_src- The total number of source nodes, avg_hops_most_src- the average distance between the gateway and most source nodes, avg_hops_all_src- the average distance between the gateway and all source nodes, largest_hop_src- the farthest distance to the gateway of all source nodes.

Every gateway adjusts the TTL of the GW_ADV message according to the following algorithm: If the total_nbr_src is less than MIN_NBR_SRC or avg_hops_most_src is less than MIN_HOPS, then the TTL is set to 0 . On the other hand, if total_nbr_src is greater than MAX_NBR_SRC or avg_hops_most_src is greater than MAX_HOPS, then, the TTL is set to the largest_hop_src, otherwise, TTL is set to the avg_hops_all_arc. Here, MIN_NBR_SRC, MIN_HOPS, MAX_NBR_SRC and MAX_HOPS are threshold values which denote minimum neighbor of sources, minimum hops, maximum neighbor of sources and maximum hops.

\section{Strategy 6:}

In this strategy [17], an approach called complete adaptive is proposed. Periodic GW_ADV messages are sent at large intervals and the periodicity is adapted whenever mobility is detected in the MANET. To decide the TTL of the GW_ADV message maximal benefit coverage algorithm [13] is used. In order to decide whether to perform adapt TTL periodicity, a heuristic function is used. At regular intervals, each gateway calculates its regulated mobility degree (RMD), which gives the amount of mobility of the source nodes registered with this gateway. The RMD parameter is used as a threshold value to determine whether to adapt the periodicity of the GW_ADV message. If an adaptation is required, it is done according to the maximal benefit coverage algorithm, as stated earlier.

\section{Strategy 7:}

This gateway discovery scheme [18] is suitable for real-time applications. It adjusts the frequency of GW_ADV messages dynamically.

The GW_ADV time interval is associated with the quality of service of the traffic. Whenever a source node chooses to send real-time traffic, if the real-time traffic is not delivered within a stipulated time period, the destination sends a QoS_LOST message back to the source. Each gateway periodically checks if it has received a QoS_LOST message in the last $\tau$ seconds from an active real-time traffic source. If it has not received, then the gateway sends a GW_ADV message. 
Otherwise, the gateway calculates the ratio of the number of real-time traffic sources which are experiencing end-to-end delay, to the total number of real-time sources using that gateway. This ratio is used as a threshold to decide whether to send a new GW_ADV message. The TTL is not adjusted dynamically in this strategy.

\section{Strategy 8:}

In this strategy [19] a load-adaptive access gateway (AG) discovery scheme is proposed which dynamically resizes the range of proactive AG advertisements and reduces $A G$ acquisition latency.

Computation of proactive area: The AG periodically broadcasts Access Gateway Advertisements (AGA) containing its load information. These advertisements are limited within $\mathrm{n}$ hop neighborhood using a TTL field. In order to adjust this area dynamically, AG should know the following information: The number of network nodes, the number of source nodes that want internet connectivity, the size of the network. It is assumed that the AG estimates the size of network and the number of nodes. The initial proactive area is calculated using the following equation.

The proactive range increases or decreases according to the network traffic which is estimated by AG during a time interval. While this strategy takes into account the load of the network while calculating the TTL value dynamically, it fails to address the problem of periodicity of GW_ADV messages.

\section{Strategy 9:}

In this strategy [20], a novel genetic algorithm is used to adjust the GW_ADV time interval, $T_{\text {MRA. The }}$ proactive gateway discovery mechanism is used for GW_ADV range. An estimation of the percentage of nodes located in the transmission range of the gateway is used to regulate the periodicity of GW_ADV message Dense neighborhood of a gateway indicates that the number of hops is more, therefore, GW_ADV messages must be sent at lengthier time intervals, resulting in higher value of periodicity $\mathrm{T}_{\mathrm{MRA}}$, in order not to congest the MANET with unnecessary control messages.

The value of $\mathrm{T}_{\mathrm{MRA}}$ is configured using a control system which uses a genetic algorithm, which works on the basis of a fitness function. The fitness function judges the suitability of the $\mathrm{T}_{\text {MRA }}$ value and is based on packet loss rate and end-to-end delay, which are perceived as the two most crucial parameters by end users. Based on the fitness function, the value of $\mathrm{T}_{\mathrm{MRA}}$ is fine tuned to reflect the changing network topology.

Strategy 10:

Maximum source coverage scheme may not be applicable to all scenarios since one part of the network may be highly loaded and other part may be lightly loaded. A new scheme called Adaptive Distributed Gateway Discovery (ADD) [21] is proposed which is based on the hypothesis that gateway advertisements should be targeted at active sources and not at other nodes. Instead of adjusting TTL at the gateway, a distributed approach is used.

To begin with TTL $=0$ and a completely reactive approach is used. A mobile node which desires Internet connectivity sends a GW_SOL message to discover a gateway. The gateway responds with GW_ADV. The GW_SOL message passes through multiple hops to reach the gateway. Each intermediate node through which it passes marks itself as an intermediate node. When the GW_ADV is sent by the gateway, it sets TTL $=1$. This message is received by mobile nodes which are one hop away from the gateway. Among these neighboring nodes, some may have marked themselves as intermediate nodes when the GW_SOL message passed through them. These nodes reset the TTL as 1 and again forward it to their neighbors. Other nodes which are not marked intermediate decrement the TTL value and throw away the message. Therefore, only intermediate nodes receive GW_ADV messages and the forward the GW_ADV by resetting TTL=1. In this way, only those active nodes desirous of maintaining connectivity with the gateway are periodically sent the GW_ADV and all other nodes are not burdened. The set of intermediate nodes is called an active region. When a node moves, its active region also moves.

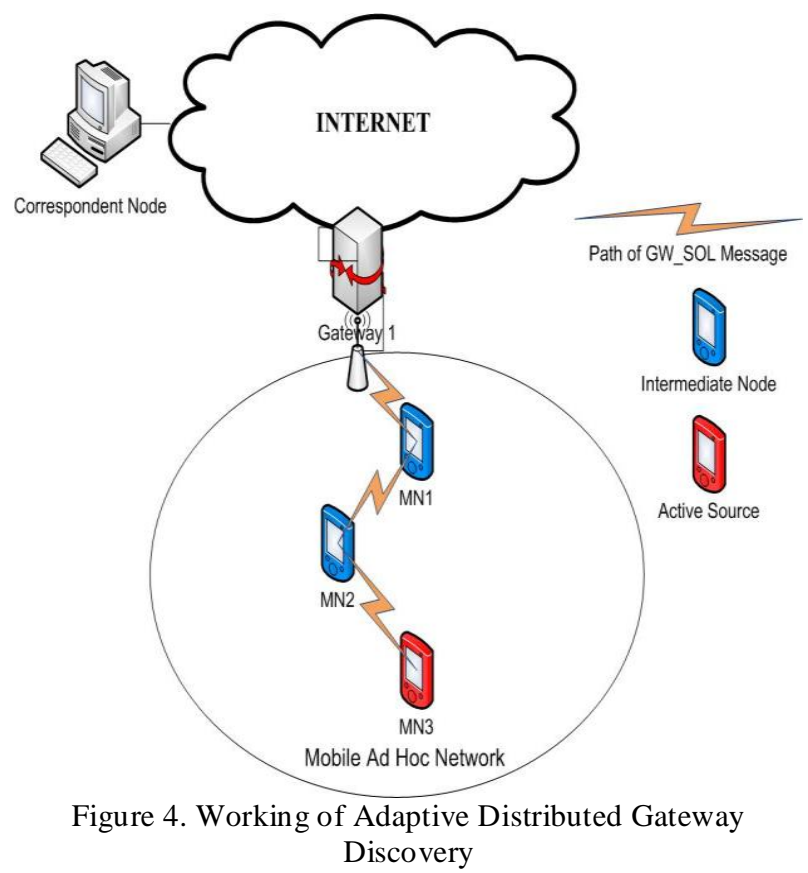

In fig 4, MN 3 solicits Internet connectivity by sending a GW_SOL message to the gateway. The set of nodes through which this GW_SOL message passes through, i.e. MN 1 and MN 2, mark themselves as intermediate nodes. When the GW_SOL message reaches the gateway, it sends GW_ADV message to its neighbor MN 1 with TTL $=1$. Now, only MN 2 resets TTL value to 1 and forwards it, since it is an intermediate node. The other gateway neighbors set TTL $=0$ and throw it away since they are not an intermediate node. 


\section{Strategy 11:}

In this strategy [22], the authors contend that the optimal TTL value depends on the particular scenario and network conditions. A futuristic architecture combining cellular and WLAN networks is proposed in which each base station or gateway decides its TTL value independently according to the link quality as well as density of active source nodes in the network.

In this strategy, a novel method of adjusting TTL value was proposed which can be an alternative to the maximal source coverage method. Periodicity of GW_ADV is not addressed.

Adjustment of the TTL value results in the proactive zone adjustment. Adjustment of the TTL value is based on the changes in average distance of active sources from the gateways and the signal strength of the gateways. Periodicity of GW_ADV messages is not addressed.

\section{Strategy 12:}

In this proposal [23], the gateway periodically generates modified router advertisement (MRA) messages each interval of time $\mathrm{T}_{\mathrm{MRA}}$. An MRA message is nothing but a GW_ADV message. These messages are propagated in a restricted area close to the gateway (proactive zone). This area is defined by the TTL value. Gateway solicitation messages generated by mobile nodes in the reactive zone are called Modified Router Solicitation (MRS) messages, which are nothing but GW_SOL messages. The optimum value for $\mathrm{T}_{\mathrm{MRA}}$ and TTL depends on the network conditions such as load etc. The objective of this work is to analyze the benefits of dynamically adjusting the $\mathrm{T}_{\mathrm{MRA}}$ and TTL values in a hybrid gateway discovery.

The scheme is based on estimating the number of Modified Router Solicitation (MRS) messages generated by the mobile nodes in an interval of time called MRSCOUNT or $\mathrm{T}_{\text {MRS }}$. Based on the $\mathrm{T}_{\mathrm{MRS}}$ messages received, the gateway predicts how many MRS requests it will receive in the next $\mathrm{T}_{\text {MRS }}$ interval.

The Maximal Source Coverage algorithm is used to set the TTL value.

\section{Strategy 13:}

There is a need for gateway discovery mechanisms that support several Heterogeneous Wireless and Mobile Networks (HWMN) applications. Recognizing this need, this strategy [24] is based on HWMN architecture. Vehicular Ad Hoc Network (VANET) is considered as a multi-hop network, as an alternative to Mobile Ad Hoc Network (MANET). In this architecture, the proposed protocol is a layer on top of the network layer. It uses channel diversity and adapts the advertisement zone of the gateways based on the location information and velocity of the mobile nodes. This mechanism is called location aided adaptation of the advertisement of the gateway.

It is assumed that the gateway recognizes the location $\mathrm{L}_{0}$ of the source vehicle $\mathrm{S}$ at time $\mathrm{t}_{0}$. The gateway can discover this information from the GW_SOL message sent by a vehicle. From the Gateway's point of view, the expected area of the source vehicle $\mathrm{S}$, which has sent the GW_SOL message, at time $t_{1}$, is the area in which $S$ is expected at time $t_{1}$. In addition, $G$ is also supposed to know the velocity of $S$ at time $t_{0}$. Then, the expected zone of $S$ at $t_{1}$ is the circle of radius $\left(t_{1-} t_{0}\right) * v$ centered at the location $\mathrm{L}_{0}$.

In this way, the gateway can adapt its proactive advertisement zone to include the source vehicle (mobile node). The periodicity of the GW_ADV message is not addressed.

\section{Strategy 14:}

A dynamic algorithm is proposed [25] based on the fuzzy system which controls the periodicity and value of the TTL. A gateway generates periodic Modified Router Advertisements (MRA) in the proactive zone. Mobile nodes outside this zone generate Modified Router Solicitation (MRS) messages. The following parameters are used:

Number of received MRS (NMRS): It is the ratio of the number of MRS messages that the sources generate and the number of active sources.

Link Changes (LC): This metric is used to measure the mobility of nodes near the gateway. It represents the ration of number of link changes a gateway detects and the number of active sources.

TTL changes (TTLC): This metric represents the ratio of number of changes in the distance of the active sources to the number of active sources.

In essence, NMRS calculates the ratio of active sources in the reactive zone and proactive zone. LC calculates the mobility of nodes near the gateway. TTLC calculates the mobility of active sources. The above three parameters are fuzzified. Based on their values, the parameters can be classified as low, moderate and high. The authors present fuzzy rules. Based on these rules, an output called convenience is produced, which can have values very low, low, moderate, high, very high. The value of the output is compared with a predetermined threshold (0.5). When it exceeds the threshold, the gateway concludes that an MRA message is necessary and broadcasts a new one. The parameters are measured each second so that the algorithm is able to adapt itself to rapid changes in the movement of nodes. The decision phase is triggered every second with updated measurements.

This algorithm only calculates the periodicity of the TTL. For value of the TTL, maximal source coverage algorithm is used.

\section{Strategy 15:}

In this strategy [26], both TTL value and periodicity of GW_ADV message are adjusted dynamically. In addition, the novelty of this strategy lies in the fact that unidirectional links are not considered while forwarding packets between mobile nodes.

To calculate the TTL value of the next GW_ADV message, the average number of hops of all source nodes is considered, as in the previous strategy. The difference between the average numbers of hops of two 
successive GW_ADV messages is added to the TTL value of the previous GW_ADV message which results in the value of the next TTL.

\section{COMPARISON FRAMEWORK OF ADAPTIVE GATEWAY DisCOVERY MECHANISMS}

In this section we provide a framework for the comparison of the Adaptive Gateway Discovery mechanisms discussed in section IV. The adaptive gateway discovery strategies can be classified into the following three types: We call the strategies which adjust the TTL value only as Type A, those which adjust the periodicity of the TTL value only as Type B, and those which adjust both the TTL value and its periodicity Type C. Fig 5 provides a classification of the adaptive gateway discovery mechanisms based on the above classification.

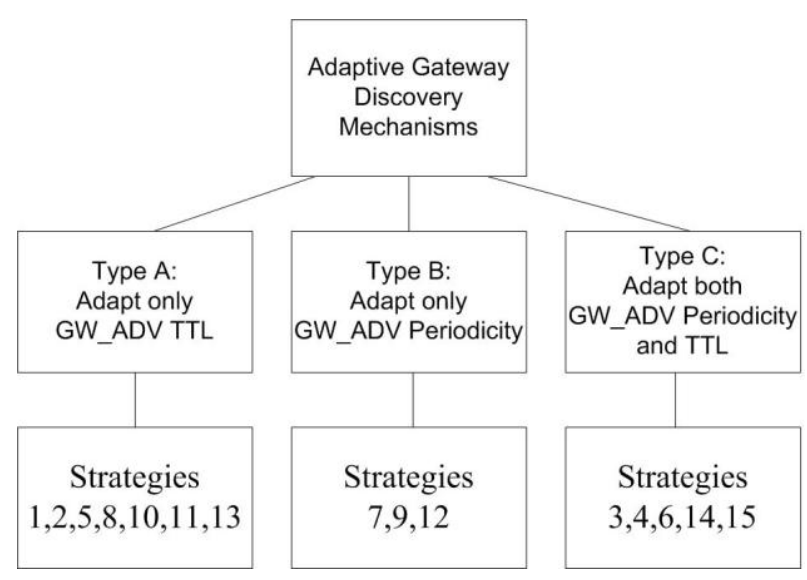

Fig 5. Classification of Adaptive Gateway Discovery Mechanisms

The adaptive gateway discovery mechanisms discussed in this paper use different parameters for adapting the GW_ADV TTL value and GW_ADV Periodicity. The parameters used by each strategy are listed in Table I. From Table I, we observe that the Maximal Source Coverage and Maximal Benefit Coverage algoritms are frequently used for GW_ADV TTL adjustment. Strategies 4 and 6 use the mobility degree to determine the GW_ADV periodicity. Only one strategy (strategy 7) uses QoS parameters for adjusting either of the two GW_ADV parameters.

Tables II, III and IV present the mobile ad hoc network routing protocol and type of architecture of Type A,B and C adaptive gateway discovery strategies. From these tables, we observe that most of the strategies use AODV routing protocol or its extended version $\mathrm{AODV}+$ for MANET routing. Almost all the strategies use the Internet integrated MANET architecture, and only two strategies (11 and 13) use a different architecture. In Table II, MUN stands for Mobile Ubiquitous Network and HWMN stands for Heterogeneous Wireless Mobile Network.
TABLE I. GW ADV TTL AND PERIODICITY PARAMETERS OF ADAPTIVE GATEWAY DISCOVERY MECHANISMS

\begin{tabular}{|c|c|c|}
\hline $\begin{array}{l}\text { Adaptive } \\
\text { Gateway } \\
\text { Discovery }\end{array}$ & $\begin{array}{c}\text { GW_ADV TTL } \\
\text { Value }\end{array}$ & GW_ADV Periodicity \\
\hline Strategy 1 & $\begin{array}{c}\text { Maximal Source } \\
\text { Coverage }\end{array}$ & ----- \\
\hline Strategy 2 & $\begin{array}{l}\text { Maximal Benefit } \\
\text { Coverage }\end{array}$ & ----- \\
\hline Strategy 3 & \multicolumn{2}{|c|}{ Average number of hops } \\
\hline Strategy 4 & $\begin{array}{c}\text { Number of hops of } \\
\text { source nodes }\end{array}$ & Movement Degree \\
\hline Strategy 5 & $\begin{array}{c}\text { Number of source } \\
\text { nodes }\end{array}$ & ----- \\
\hline Strategy 6 & $\begin{array}{l}\text { Maximal Benefit } \\
\text { Coverage }\end{array}$ & $\begin{array}{l}\text { Regulated mobility degree } \\
\text { (RMD) }\end{array}$ \\
\hline Strategy 7 & ----- & $\begin{array}{l}\text { QoS parameters(End-to- } \\
\text { End delay) }\end{array}$ \\
\hline Strategy 8 & $\begin{array}{c}\text { Network size, } \\
\text { number of nodes } \\
\text { and data packet } \\
\text { size }\end{array}$ & ----- \\
\hline Strategy 9 & ------ & $\begin{array}{c}\text { Genetic algorithm based } \\
\text { on packet-loss-rate and } \\
\text { end-to-end delay }\end{array}$ \\
\hline Strategy 10 & $\begin{array}{l}\text { Calculated at each } \\
\text { hop }\end{array}$ & ----- \\
\hline Strategy 11 & $\begin{array}{l}\text { Signal quality, } \\
\text { average hops to } \\
\text { active sources }\end{array}$ & ----- \\
\hline Strategy 12 & ----- & $\begin{array}{c}\text { Estimating number of } \\
\text { MRS messages }\end{array}$ \\
\hline Strategy 13 & $\begin{array}{l}\text { Location and } \\
\text { velocity of mobile } \\
\text { node }\end{array}$ & ------ \\
\hline Strategy 14 & $\begin{array}{c}\text { Maximal Source } \\
\text { Coverage }\end{array}$ & $\begin{array}{l}\text { Fuzzy mechanism based } \\
\text { on NMRS, LC, TTLC }\end{array}$ \\
\hline Strategy 15 & \multicolumn{2}{|c|}{ Average number of hops of all source nodes } \\
\hline
\end{tabular}

TABLE II. COMPARISON OF TYPE A STRATEGIES
\begin{tabular}{|l|l|l|}
\hline $\begin{array}{c}\text { Adaptive } \\
\text { Gateway } \\
\text { Discovery }\end{array}$ & $\begin{array}{c}\text { MANET } \\
\text { Routing } \\
\text { Protocol }\end{array}$ & $\begin{array}{c}\text { Type of } \\
\text { Architecture }\end{array}$ \\
\hline Strategy 1 & AODV & IIM \\
\hline Strategy 2 & AODV & IIM \\
\hline Strategy 5 & AODV & IIM \\
\hline Strategy 8 & AODV+ & IIM \\
\hline Strategy 10 & AODV+ & IIM \\
\hline Strategy 11 & DSR & MUN \\
\hline Strategy 13 & LAGAD & HWMN \\
\hline
\end{tabular}

TABLE III. COMPARISON OF TYPE B STRATEGIES

\begin{tabular}{|c|c|c|}
\hline $\begin{array}{c}\text { Adaptive } \\
\text { Gateway } \\
\text { Discovery }\end{array}$ & $\begin{array}{c}\text { MANET } \\
\text { Routing } \\
\text { Protocol }\end{array}$ & $\begin{array}{c}\text { Type of } \\
\text { Architecture }\end{array}$ \\
\hline Strategy 7 & AODV & IIM \\
\hline Strategy 9 & AODV+ & IIM \\
\hline Strategy 12 & AODV+ & IIM \\
\hline
\end{tabular}

TABLE IV. COMPARISON OF TYPE C STRATEGIES

\begin{tabular}{|l|l|l|}
\hline $\begin{array}{c}\text { Adaptive } \\
\text { Gateway } \\
\text { Discovery }\end{array}$ & $\begin{array}{c}\text { MANET } \\
\text { Routing } \\
\text { Protocol }\end{array}$ & $\begin{array}{c}\text { Type of } \\
\text { Architecture }\end{array}$ \\
\hline Strategy 3 & AODV & IIM \\
\hline Strategy 4 & AODV+ & IIM \\
\hline Strategy 6 & AODV & IIM \\
\hline Strategy 14 & AODV+ & IIM \\
\hline Strategy 15 & AODV & IIM \\
\hline
\end{tabular}




\section{CONCLUSION}

In this paper, we have presented a survey of adaptive gateway discovery mechanisms in heterogeneous networks. We have classified the adaptive gateway discovery mechanisms into three types based on the parameters GW_ADV periodicity and TTL. We summarize that an ideal adaptive gateway discovery mechanism must address both the issues of dynamically adjusting the TTL value as well as the periodicity of GW_ADV messages. Such a strategy must take into account network conditions like traffic load and node density, as well as signal strength. The adaptive gateway discovery strategy must not only address Internet integrated MANET, but must also work with more heterogeneous architectures envisage in future $4 \mathrm{G}$ networks [27].

\section{REFERENCES}

[1] C.S.R. Murthy, B.S. Manoj, "Ad Hoc Wireless Networks, Architectures and Protocols", Pearson Education, 2004.

[2] E.M. Royer, C-K. Toh, "A Review of Current Routing Protocols for Ad Hoc Mobile Wireless Networks", IEEE Personal Communications Magazine, 46-55, 1999.

[3] Khaleel Ur Rahman Khan, Rafi U Zaman, and A. Venugopal Reddy, "Performance Comparison of On-Demand and Table Driven Ad Hoc Routing Protocols using NCTUns", 10th International Conference UKSIM Cambridge England, 336-341, 2008.

[4] C.E. Perkins, "Mobile Networking Through Mobile IP”, IEEE Internet Computing, Vol. 2, Issue 1, 5869, 1998.

[5] Wakikawa, Ryuji, Jari T. Malinen, Charles E. Perkins, Anders Nilsson, and Antti J. Tuominen. "Global connectivity for IPv6 mobile ad hoc networks." Internet Engineering Task Force, Internet Draft, 2003

[6] Rafi U Zaman, Khaleel Ur Rahman Khan, A. Venugopal Reddy, "A Review of Gateway Load Balancing Strategies in Integrated InternetMANET", IEEE International Conference on Internet Multimedia Services Architecture and Applications (IMSAA), 1-6 2009.

[7] C. Ahlund and A. Zaslavsky, "Multihoming with Mobile IP", High-Speed Networks and Multimedia Communucations (LNCS), 235-243, 2004.

[8] Jing Nie, Liaoyuan Zeng, Jiangchua Wen "Handoffs in Integrating Ad Hoc Networks to Internet", Proceedings of the 2007 International Conference on Convergence Information Technology, 1378-1377, 2007.

[9] Khaleel Ur Rahman Khan, Rafi U Zaman and A. Venugopal Reddy, “An Analytical Framework for the Assessment of Mobile IP Overhead involved in the Integrated Internet-MANET", International
Journal of Interactive Mobile Technologies (iJIM), Vol 4, No 1, 22-33, (2010).

[10] Ghassemian, M.; Hofmann, P.; Prehofer, C.; Friderikos, V.; Aghvami, H., "Performance analysis of Internet gateway discovery protocols in ad hoc networks," Wireless Communications and Networking Conference. WCNC. 2004 IEEE, Vol.1, 21-25, 2004.

[11] Ali Hamidian, Ulf Körner, Anders Nilsson: "Performance of Internet Access Solutions in Mobile Ad hoc Networks", Lecture Notes in Computer Science (LNCS), vol. 3427, 189-201, 2004.

[12] Pedro M. Ruiz, Antonio F. Gomez-Skarmeta: "Maximal Source Coverage Adaptive Gateway Discovery for Hybrid Ad Hoc Networks", Lecture Notes in Computer Science, vol.3158, 28-41, 2004.

[13] Pedro M. Ruiz, Antonio F. Gomez-Skarmeta: "Enhanced Internet Connectivity for Hybrid Ad hoc Networks Through Adaptive Gateway Discovery", 29th Annual IEEE International Conference on Local Computer Networks, 370-377, 2004.

[14] S. Bin, S. Bingxin, L. Bo, H. Zhonggong, and Z. Li, "Adaptive gateway Discovery Scheme for Connecting Mobile Ad Hoc Networks to the Internet," Proceedings of International Conference on Wireless Communications, Networking and Mobile Computing, vol. 2, 795-799, 2005.

[15] Kaijie Zhang; Yong Xiang; Meilin Shi; "Adaptive Internet Gateway Discovery Scheme for Mobile Ad Hoc Networks," First International Conference on Communications and Networking in China, 2006. ChinaCom '06., 1-5, 25-27, 2006.

[16] Rakeshkumar, V.; Misra, M.; "An Efficient Mechanism for Connecting MANET and Internet through Complete Adaptive Gateway Discovery," First International Conference on Communication System Software and Middleware, (Comsware 2006). 1-5, 2006.

[17] Shen Bin; Ke Haiyan; Hu Zhonggong; , "Adaptive Mechanisms to Enhance Internet Connectivity for Mobile Ad Hoc Networks," Wireless Communications, Networking and Mobile Computing, 2006. WiCOM 2006.International Conference on , 1-4, 22-24 Sept. 2006

[18] Mari Carmen Domingo, Rui Prior; “An Adaptive Gateway Discovery Algorithm to support QoS When Providing Internet Access to Mobile Ad Hoc Networks", Journal of Networks, Vol 2, No 2 (2007), 33-44, 2007

[19] Bok-Nyong Park, Wonjun Lee, Choonwa Lee, "QoS-aware Internet access schemesfor wireless ad hoc networks", Computer Communications 30 (2007), 369-384, (2007).

[20] A.J. Yuste, F.D. Trujillo,Alicia Trivino, E. Casilari; "An adaptive gateway discovery for mobile ad hoc networks", $5^{\text {th }}$ ACM International workshop on mobility management and wireless access, 159-162, 2007. 
[21] Javaid, U., Rasheed, T.M., Meddour, D., Ahmed, T.: Adaptive Distributed Gateway Discovery in Hybrid Wireless Networks. WCNC - 2008, 27352740. (2008)

[22] Fang Xie, Lei Du, Yong Bai, Lan Chen, "Adaptive Gateway Discovery Scheme for Mobile Ubiquitous Networks", WCNC 2008, 2916-2020, 2008.

[23] Triviño-Cabrera, A., Ruiz-Villalobos, B., Casilari, E., Yuste-Delgado, A.J.: Study on the need for adaptive gateway discovery in MANETs. In IWCMC (2009) 1091-1095. (2009)

[24] Boukerche, A.; Abrougui, K.; Pazzi, R.W.; "An Efficient Hybrid Adaptive Location-Aided Gateway Advertisement and Discovery Protocol for Heterogeneous Wireless and Mobile Networks," Global Telecommunications Conference, 2009. GLOBECOM 2009. IEEE, vol., no., pp.1-6, Nov. 30 2009-Dec. 42009

[25] A.J. Yuste, Alicia Trivino, F.D. Trujillo, E. Casilari: "Using Fuzzy Logic in Hybrid Multihop Wireless Networks". International Journal of Wireless \& Mobile Networks Volume 2, Issue 3, 96-108, 2010.

[26] Lin Zhuang, Yuan-an Liu, Kai-ming Liu, Lin-bo Zhai, Ming Yang, "An adaptive algorithm for connecting mobile ad hoc network to Internet with unidirectional links supported", The Journal of China Universities of Posts and Telecommunications, Volume 17, Supplement 1, July 2010, Pages 44-49, 2010.

[27] Dave Cavalcanti, Carlos Cordeiro, Dharma P. Agrawal, B. Xie, and Anup Kumar, "Issues in Integrating Cellular Networks, WLANs, and MANETs: A Futuristic Heterogeneous Wireless Network," IEEE Wireless Communications Magazine, Special issue on Toward Seamless Internetworking of Wireless LAN and Cellular Networks, Vol. 12, No. 3, 30-41, 2005.

[28] Fekri M. Abduljalil, Shrikant K. Bodhe, "A survey of integrating IP mobility protocols and mobile ad hoc networks". IEEE Communications Surveys and Tutorials 9 (1-4): 14-30, 2007.

[29] Khaleel Ur Rahman Khan, Rafi U Zaman, A.Venugopal Reddy, "Integrating Mobile Ad Hoc Networks and the Internet: challenges and a review of strategies", Communication Systems Software and Middleware and Workshops, COMSWARE 2008. 3rd International Conference, 536 -543, 2008.

[30] Shuo Ding, "A survey on integrating MANETs with the Internet: Challenges and designs". Computer Communications 31, Elsevier, 35373551 (2008)

[31] Rakesh Kumar, Anil K. Sarje, and Manoj Misra, "Review Strategies and Analysis of Mobile Ad Hoc Network- Internet Integration Solutions", IJCSI International Journal of Computer Science Issues, Vol. 7, Issue 4, No 6, July 2010.

[32] Khaleel Ur Rahman Khan, Rafi U Zaman and A. Venugopal Reddy, “An Analytical Framework for the Assessment of Mobile IP Overhead involved in the Integrated Internet-MANET', International Journal of Interactive Mobile Technologies (iJIM), Vol 4, No 1 (2010) doi:10.3991/ijim.v4i1.1027, pages 22-33, 2010.

\section{Authors Biography}

Rafi U Zaman was born in Hyderabad, India, on May 19, 1979. He obtained the B.Sc. (Computer Science), M.C.A. and M.Tech (CSE) degrees from Osmania University in 1999, 2002 and 2009 respectively. He is currently working as Associate Professor in Muffakham Jah College of Engineering And Technology, Hyderabad and pursuing Ph.D. in the area of efficient integration of wired and wireless networks, from Osmania University.

Dr. Khaleel Ur Rahman Khan obtained B.E. (CSE) from Osmania University in 1993 and M.Tech (CS) from JNTU in 1998 and Ph.D. from Osmania University in 2009. He is presently working as Professor in the CSE Department and Dean (academics) at Ace Engineering College, Hyderabad. His research interests include Heterogeneous Networks and Data Mining.

Dr. A. Venugopal Reddy has done his M.Tech. from IIT Delhi in 1979 and Ph.D. from University of Roorkee (IIT Roorkee) in the year 1994. He has a total of 34 years of teaching experience, teaching to both undergraduate and graduate students. Currently he is working as Professor in Computer Science and Engineering Department at the College of Engineering, Osmania University. His current subject interests include Parallel Algorithms, Computer Networks, Mobile Ad hoc Networks. 